\title{
Small-Molecule CD4-Mimics: Structure-Based Optimization of HIV-1 Entry Inhibition
}

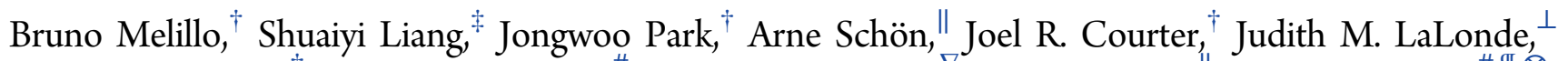
Daniel J. Wendler, ${ }^{\dagger}$ Amy M. Princiotto, ${ }^{\#}$ Michael S. Seaman, ${ }^{\nabla}$ Ernesto Freire, ${ }^{\|}$Joseph Sodroski, ${ }^{\#, I I, \otimes}$ Navid Madani, ${ }^{\#}$ Wayne A. Hendrickson, ${ }^{\ddagger, \S}$ and Amos B. Smith, III*, ${ }^{\dagger}$

${ }^{\dagger}$ Department of Chemistry, University of Pennsylvania, Philadelphia, Pennsylvania 19104, United States

${ }^{\ddagger}$ Department of Biochemistry and Molecular Biophysics and ${ }^{\S}$ Department of Physiology and Cellular Biophysics, Columbia University, New York, New York 10032, United States

"Department of Biology, The Johns Hopkins University, Baltimore, Maryland 21218, United States

${ }^{\perp}$ Department of Chemistry, Bryn Mawr College, Bryn Mawr, Pennsylvania 19010, United States

${ }^{\#}$ Department of Cancer Immunology and Virology, Dana-Farber Cancer Institute, ${ }^{\nabla}$ Center for Virology and Vaccine Research, Beth Israel Deaconess Medical Center, and ${ }^{\mathrm{TI}}$ Department of Microbiology and Immunobiology, Harvard Medical School, Boston, Massachusetts 02115, United States

${ }^{\otimes}$ Department of Immunology and Infectious Diseases, Harvard School of Public Health, Boston, Massachusetts 02115, United States

\section{Supporting Information}

ABSTRACT: The optimization, based on computational, thermodynamic, and crystallographic data, of a series of small-molecule ligands of the Phe43 cavity of the envelope glycoprotein gp120 of human immunodeficiency virus (HIV) has been achieved. Importantly, biological evaluation revealed that the small-molecule CD4 mimics (4-7) inhibit HIV-1 entry into target cells with both significantly higher potency and neutralization breadth than previous congeners, while maintaining high selectivity for the target virus. Their binding mode was characterized via thermodynamic and crystallographic studies.

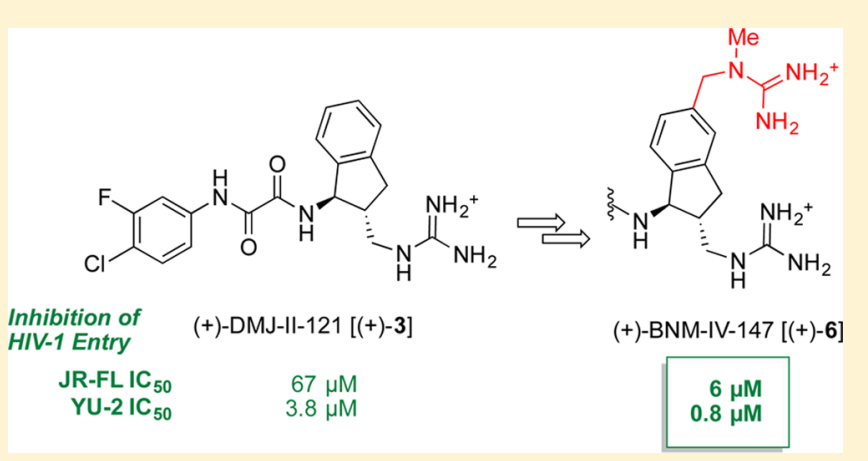

KEYWORDS: HIV, gp120, CD4, entry inhibitor, structure-based drug design, thermodynamics, X-ray crystallography, viral inhibition, protein-protein interactions
W ith an estimated 35 million people currently living with HIV-1 worldwide in conjunction with 2.1 million new infections each year, ${ }^{1}$ the development of effective new strategies to achieve both prevention of HIV transmission, complementing known pre-exposure prophylactics ${ }^{2,3}$ and depletion of the "viral reservoir" in HIV-infected patients remain critically important challenges.

Recently, we reported the structure-based design, synthesis, and evaluation of the small-molecule CD4-mimic DMJ-II-121 (3), a micromolar inhibitor of HIV-1 $1_{\mathrm{YU}-2}$ entry into human immune cells. ${ }^{4,5}$ Like the initial congeners reported by Debnath et al. (see 1 and 2, Figure 1),,$^{6-8} 3$ binds the Phe 43 cavity of the viral envelope (Env) glycoprotein gp120, blocking CD4 binding and triggering a conformational change that ultimately results in viral inactivation.

A particularly attractive feature of this mechanism of action resides in the opportunity to elicit immune responses specific to epitopes that become exposed on the surface on Env upon the binding of CD4 mimics. Importantly, we have demonstrated that antibodies can indeed recognize gp120 bound to the CD4 small-molecule mimics, both on the surface of the virion ${ }^{9}$ and, more recently, on the surface of infected cells. ${ }^{10}$ Thus, in our efforts to enhance the direct inhibitory activity of the smallmolecule CD4 mimetics, we have sought to improve our understanding of the underlying molecular mechanisms. Logically, we chose 3 as the starting point for the design of a new generation of more potent small-molecule entry inhibitors.

Having previously conducted structural optimization on Regions I and II of the parent scaffold, defined respectively by the halogenated aromatic ring and the oxalamide linker (Figure 2), our efforts focused on modification of the substituted indane ring comprising Region III. ${ }^{11}$ Importantly, the protein:ligand cocrystal structure of 3 permitted definition of potential binding hotspots in proximity to the aromatic portion of the indane. ${ }^{4}$ Based on the structure and "hotspot" analysis, ${ }^{12}$ we also demonstrated that a methylene (one carbon) spacer

Received: December 9, 2015

Accepted: January 13, 2016

Published: January 19, 2016 

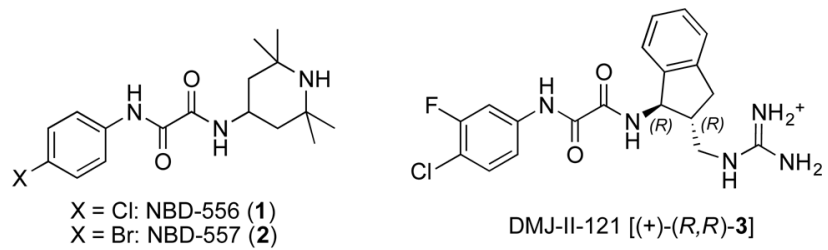

DMJ-II-121 [(+)-(R,R)-3]
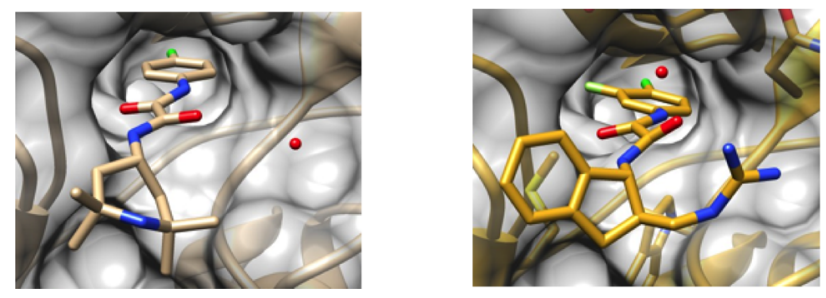

Figure 1. NBD-556 (1), (+)-DMJ-II-121 $[(+)-(R, R)-3]$, and their gp120-bound X-ray structures (PDB accession codes: 3TGS and 4I53, respectively).

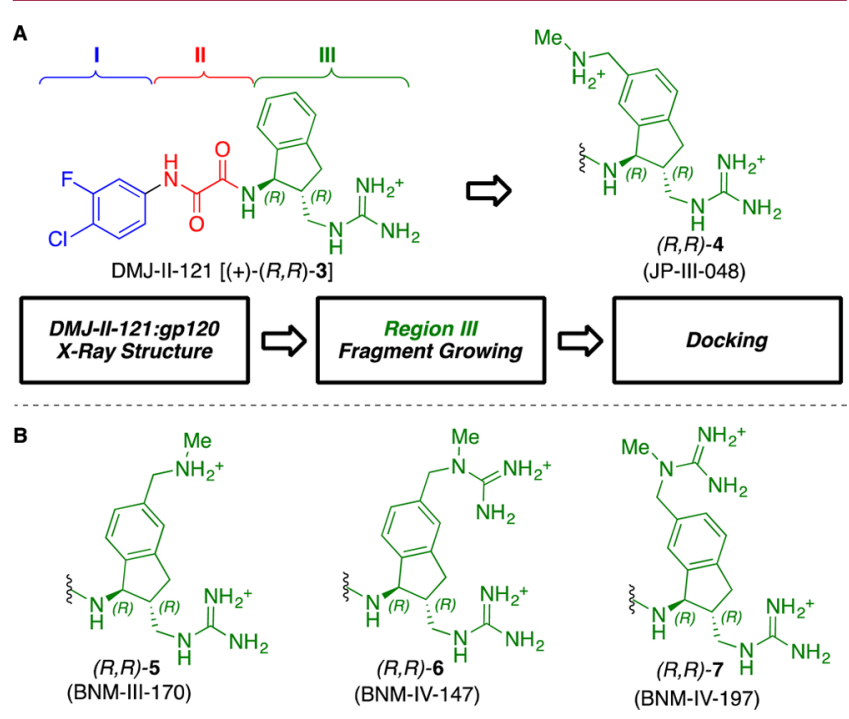

Figure 2. (A) Computational design of 4. (B) Further evolution of inhibitor Region III scaffolds.

present between the indane skeleton and the guanidinium moiety in 3 proved optimal for viral entry inhibition; ${ }^{4}$ therefore, no modifications were planned in that section of Region III. Instead, a virtual fragment growing algorithm, ${ }^{13}$ followed by docking, ${ }^{14,15}$ was carried out to establish contacts between the potentially new analogues and "hotspot" ${ }^{\mathrm{gp}}{ }^{120} \mathrm{Gly} 472$, a highly conserved gp120 residue located in the outer domain near the vestibule of the Phe43 cavity, associated with moderate Env restructuring (i.e., allosteric signal) upon CD4 binding. ${ }^{12,16}$ This computational analysis suggested that addition of a (methylamino)methyl unit at position 6 of the indane skeleton (see 4, Figure 2) might result in hydrogen bonding with the backbone carbonyl of ${ }^{\mathrm{gp}}{ }^{120}$ Gly 472 . Our computational model also predicted binding between the guanidinium terminus and ${ }_{\mathrm{gp}}{ }^{120}$ Asp 368 , an interaction that had been predicted by our prior modeling work, but to date had proven elusive. ${ }^{5}$

To evaluate in rapid fashion the biological impact of this previously unexplored scaffold substitution pattern, the synthesis of the (methylamino)methyl-bearing analogue JP-III-048 (4) was carried out initially in racemic form (see Supporting Information). Pleasingly, a viral infectivity assay employing $( \pm)-4$ revealed that the proposed structural modification did not result in significant loss of inhibitory activity relative to 3 $\left(\mathrm{HIV}-1_{\mathrm{YU}-2} \mathrm{IC}_{50} 6.8 \mu \mathrm{M}\right)$. To determine the effect of absolute stereoconfiguration on bioactivity, enantioenriched (+)- and (-)-4 were prepared by separation of the ultimate synthetic intermediate via semipreparative supercritical fluid chromatography (SFC) employing a chiral stationary phase. Global deprotection resulted in (+)- and (-)-4 (in $>96 \%$ ee). Evaluation of the individual enantiomers revealed that similar to the previous enantiopure congeners, the $(R, R)$-stereoisomer (+)-4 exhibited 40-fold stronger inhibitory activity than the $(S, S)$-counterpart (YU-2 $\mathrm{IC}_{50} 2.1$ and $87.8 \mu \mathrm{M}$, respectively). This result represents a gain, albeit modest, relative to $(+)-3$ (YU-2 $\mathrm{IC}_{50} 3.8 \mu \mathrm{M}$ ). We were, however, pleased to find that the binding affinity of (+)-4 to full-length gp120, measured by isothermal titration calorimetry (ITC), had increased quite significantly $\left[K_{\mathrm{D}} 24\right.$ vs $110 \mathrm{nM}$ for $\left.(+)-3\right]$.

Given the difference in activity between $(+)$ - and (-)-4, the original synthetic route was modified to permit facile preparation of the single enantiomers of this new generation of entry inhibitors. To this end, the enantioselective synthesis of (+)-4 began with protection of commercially available 6bromoindanone $(8)$ as the corresponding dioxolane, followed by lithiation and formylation, to furnish aldehyde 9 (Scheme 1 ). Reductive amination, protection of the resulting secondary

Scheme 1. Enantioselective Synthesis of $(+)-(R, R)-4$

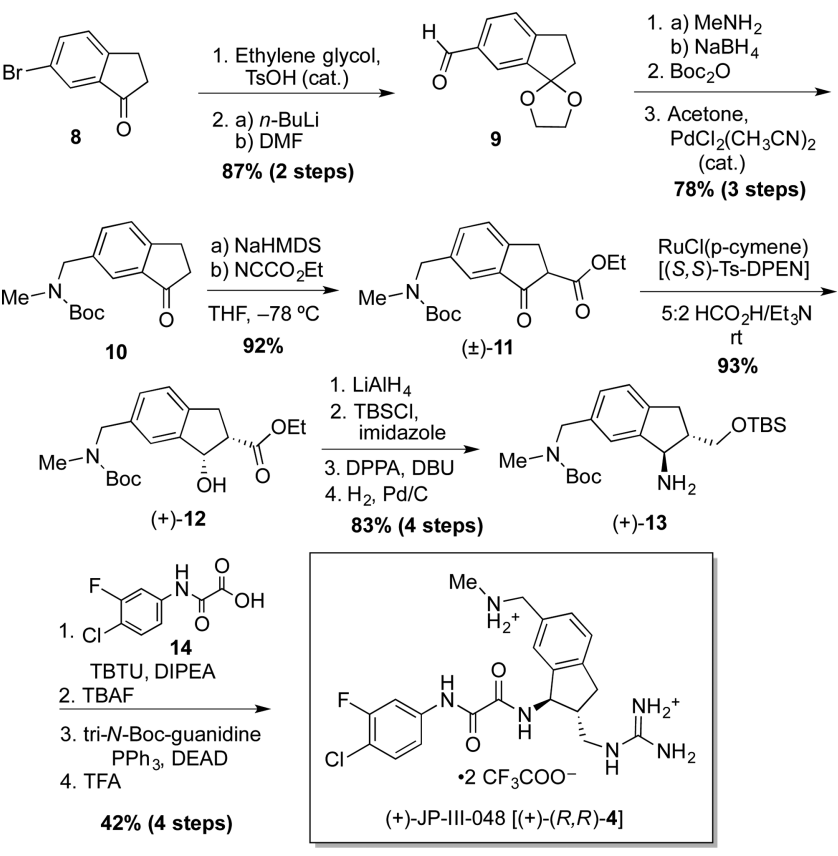

amine as the corresponding Boc carbamate, and selective removal of carbonyl protection then revealed indanone 10, now bearing the desired (methylamino)methyl functionality. Ketone 10 was next carboxylated at the $\alpha$-position, and the resulting racemic ketoester $( \pm)-11$ subjected to dynamic-kinetic resolution employing chiral ruthenium catalyst $\mathrm{RuCl}(p$ cymene) $[$ Ts-DPEN $],{ }^{19}$ which permitted transfer hydrogenation with excellent stereoselectivity (er 99:1), as demonstrated by chiral phase SFC. This transformation was carried out employing both enantiomers of the ruthenium catalyst, thus permitting access to both enantiomers of $\beta$-hydroxyester 12 . Each enantiomer was then taken through the following identical sequence. Exhaustive reduction of $(+)-12$, initially 
Table 1. Small-Molecule CD4Mimics: Inhibition of Viral Entry and Protein-Ligand Affinity

\begin{tabular}{|c|c|c|c|c|c|c|c|c|c|}
\hline \multirow{2}{*}{\multicolumn{2}{|c|}{ Compound }} & \multicolumn{4}{|c|}{ Viral Entry Inhibition $\left(\mathrm{IC}_{50}, \mu \mathrm{M}\right)$} & \multicolumn{4}{|c|}{ Binding Thermodynamics ${ }^{\mathrm{c}}$} \\
\hline & & JR-FL ${ }^{\mathrm{a}}$ & $\mathbf{Y U}-\mathbf{2}^{\mathbf{a}}$ & AD8 $^{\mathrm{a}}$ & A-MLV & $\mathbf{K}_{\mathbf{D}}(\boldsymbol{\mu} \mathbf{M})$ & $\Delta \mathbf{G}^{\mathbf{d}}$ & $\Delta \mathbf{H}^{\mathrm{d}}$ & $-\mathbf{T} \Delta \mathbf{S}^{\mathbf{d}}$ \\
\hline \multirow[b]{5}{*}{$(R, R)$-series } & $(+)-3$ & $66.8 \pm 9.5$ & $3.8 \pm 1.3$ & $8.5 \pm 2.1$ & $94.3 \pm 5.7$ & 0.11 & -9.5 & -17.9 & +8.4 \\
\hline & $(+)-4$ & $37.3 \pm 13.8$ & $2.1 \pm 0.9$ & $6.7 \pm 0.6$ & $>100$ & 0.024 & -10.4 & -28.2 & +17.8 \\
\hline & $(+)-5$ & $14.5 \pm 1.5$ & $1.3 \pm 0.2$ & $3.1 \pm 1.7$ & $>100$ & 0.047 & -10.0 & -20.7 & +10.7 \\
\hline & $(+)-6$ & $6.1 \pm 0.5$ & $0.8 \pm 0.1$ & $1.6 \pm 0.9$ & $>100$ & 0.095 & -9.6 & -15.4 & +5.8 \\
\hline & $(-)-7$ & $19.7 \pm 9.9$ & $1.6 \pm 0.4$ & 1.2 & $83.7 \pm 16.4$ & 0.095 & -9.6 & -14.2 & +4.6 \\
\hline \multirow{2}{*}{$(S, S)$-series } & $(-)-4$ & $>100$ & $87.8 \pm 12.2$ & $>100$ & $>100$ & 3.4 & -7.5 & -11.3 & +3.8 \\
\hline & $(-)-5$ & $>100$ & 21 & -- & $>100$ & 1.2 & -8.1 & -13.2 & +5.1 \\
\hline
\end{tabular}

${ }^{a_{T}}$ The $\mathrm{IC}_{50}$ was determined in Cf2Th-CD4/CCR5 cells infected with HIV-1 virus of the strains indicated. ${ }^{b}$ The IC 50 in cells infected with HIV-1 pseudotyped with the envelope glycoproteins of amphotropic murine leukemia virus (A-MLV). ${ }^{c}$ As deemed by ITC employing YU-2 gp120. ${ }^{d}$ Energies in $\mathrm{kcal} / \mathrm{mol}$.

utilizing sodium borohydride in methanol, resulted in partial epimerization (cis/trans $\approx 3: 1$ ). Pleasingly, treatment of $(+)-12$ with the more reactive lithium aluminum hydride in an aprotic solvent (THF) provided the desired diol as a single stereoisomer. Selective silylation of the primary hydroxyl followed by treatment with diphenylphosphorylazide provided the corresponding azide with complete inversion of stereochemistry at the benzylic position. Palladium-catalyzed hydrogenation next furnished amine $(+)-13$. Following union with oxalic acid 14, fluoride-mediated removal of the silyl group furnished the corresponding primary alcohol, which in turn was subjected to Mitsunobu conditions ${ }^{18}$ to install the requisite guanidine functionality and reveal, after treatment with trifluoroacetic acid, target inhibitor $(+)-(R, R)-4$ as the corresponding bis(trifluoroacetate) salt. Gratifyingly, the synthetic route proved highly efficient, furnishing the target compound in $20 \%$ yield over 15 steps from commercially available 6-bromoindanone (8, Scheme 1). The alternate enantiomer $(-)-(S, S)-4$ was synthesized in identical manner from $(-)-12$.

To understand the structural basis of the increased bioactivity, (+)-(R,R)-4 was soaked into preformed crystals of gp120 from HIV Clade C1086, ${ }^{19}$ and diffraction data was obtained at $2.7 \AA ̊$ Bragg spacings (see Supporting Information). The resulting protein:ligand structure revealed that, compared to $(+)-(R, R)-3$ entry inhibitor, (+) $-(R, R)-4$ establishes two additional hydrogen-bonding interactions, one with the backbone carbonyl of ${ }^{9 p 120} \mathrm{Gly} 431$ and another with the carbonyl of ${ }^{\mathrm{gp}}{ }^{120} \mathrm{Gly} 472$, in agreement with our computational prediction. Disappointingly, however, $(+)-(R, R)-4$ failed to bind ${ }^{\mathrm{gp} 120}$ Asp368. Instead, as in the case of $(+)-(R, R)-3,{ }^{4}$ the guanidinium terminus interacts with the backbone carbonyl of ${ }_{\mathrm{gp}}{ }^{120}$ Met426. Considering (i) the diverse nature of the hotspots located in proximity to the Phe43 cavity (i.e., their varying contributions to the global allosteric signal upon binding of CD4), and (ii) the proximity of the ligand's guanidinium to the still elusive ${ }^{g p 120} A s p 368$, we reasoned that subtle structural modifications resulting in changes in ligand orientation might significantly impact bioactivity. We thus set out to prepare analogues now bearing the (methylamino)methyl moiety at indane position 5 (see 5, Figure 2). The route described above, in this case employing commercially available 5-bromoindanone (see Supporting Information for details) in similar synthetic fashion, readily furnished the target compounds $(+)-(R, R)$ - and $(-)-(S, S)-5$ (Figure 2).

Although $(+)-(R, R)-5$ displayed only a modest improvement in activity against HIV-1 monotropic strains YU2 and ADA (clone $\mathrm{AD} 8$ ) ( $\mathrm{IC}_{50} 1.5$ and $6.4 \mu \mathrm{M}$, respectively), a 5-fold increase in potency relative to congener 3 was observed against strain JR-FL ( $\left.\mathrm{IC}_{50} 14.2 \mu \mathrm{M}\right)$. Again, however, (-)-(S,S)-5 displayed a lower level of viral entry inhibition (JR-FL $\mathrm{IC}_{50}>$ $100 \mu \mathrm{M}$, Table 1). The neutralization breadths of the more potent CD4 mimics, specifically $(+)-(R, R)-4$ and $(+)-(R, R)-5$, were measured against a panel of $52 \mathrm{HIV}-1$ Env pseudoviruses. Like for previous congeners, ${ }^{4}$ clade $\mathrm{B}$ pseudoviruses were the most sensitive to the new inhibitors (9/9 strains were neutralized), with also significant activity in clades $C(11 / 17)$ and $\mathrm{D}(3 / 3)$. While both assayed compounds demonstrated similar inhibitory profiles, $(+)-(R, R)-5$ exhibited 1.3 -to-1.8-fold lower geometric mean titer (GMT) inhibitory concentrations than (+)-(R,R)-4 in clades $\mathrm{B}$ (GMT $\left.\mathrm{IC}_{50} 3.5 \mu \mathrm{M}\right)$ and $\mathrm{C}$ (GMT $\mathrm{IC}_{50} 17 \mu \mathrm{M}$ ), thus corroborating the trend observed with individual strains (see Supporting Information for further details).

However, when we turned to ITC to evaluate the binding affinities of these new analogues, we discovered that, although more active against viral entry, $(+)-(R, R)-5$ binds monomeric gp120 with a lower affinity than $(+)-(R, R)-4\left(K_{\mathrm{D}} 47\right.$ vs $\left.24 \mathrm{nM}\right)$. Interestingly, compared to the immediate predecessor, $(+)-(R, R)-5$ elicits a lowered entropic penalty $(-T \Delta S=$ $+10.7 \mathrm{kcal} / \mathrm{mol}$ vs $-T \Delta S=+17.8 \mathrm{kcal} / \mathrm{mol}$, Table 1$)$. The difference in thermodynamic signatures between $(+)-(R, R)-4$ and $(+)-(R, R)-5$ upon binding gp120 suggests that subtle structural differences (e.g., in this case, position isomerism) could be responsible for inducing significantly dissimilar binding modes, a hypothesis we again sought to evaluate crystallographically.

The resulting structure of gp120-bound (+)-(R,R)-5, obtained employing the same technique as described above (see Supporting Information), revealed a generally similar binding pose to that of $(+)-(R, R)-4$. Orientations for the indane rings differ slightly such that the substituent projects into much of the same space despite being moved from position 5 to position 6. Nevertheless, the hydrogen bond from the (methyamino) methyl group to the carbonyl of ${ }^{8 p}{ }^{120} \mathrm{Gly} 472$ is now lost $\left[d_{\mathrm{N}-\mathrm{O}}=3.65 \AA\right.$ in 5 vs $3.08 \AA$ in 4$]$. This change may 
explain the decreased enthalpic contribution to the binding energy (Table 1). The observations derived from the structure of $(+)-(R, R)-5$ :gp120 with perhaps more immediate practical implications was the proximity of the ligand's (methylamino)methyl side-chain to ${ }^{\mathrm{gp}}{ }^{120}$ Asp474, a CD4 binding hotspot associated with a purely enthalpic signal. ${ }^{12}$ To exploit this feature we directly converted the secondary amine in $(+)-(R, R)-5$ to a guanidinium moiety, which would in effect extend the reach of the side-chain and in turn potentially establish a strong interaction with the nearby carboxylate of ${ }^{\mathrm{gp}}{ }^{120}$ Asp474. Pleasingly, selective incorporation of the new guanidine functionality could be readily achieved in one step by subjecting $(+)-(R, R)-5$ to $1 H$-pyrazol-1-carboxamidine hydrochloride, to furnish $(+)-(R, R)-6$ (see Supporting Information). To our delight, $(+)-(R, R)-6$ inhibited infection of HIV-1 at lower concentrations than all previous analogues in all tested strains (Figure 3; ${ }^{\mathrm{YU}-2} \mathrm{IC}_{50} 0.8 \mu \mathrm{M} ;{ }^{\mathrm{JR}-\mathrm{FL}} \mathrm{IC}_{50} 6.2 \mu \mathrm{M} ;{ }^{\mathrm{AD} 8} \mathrm{IC}_{50} 3.3$

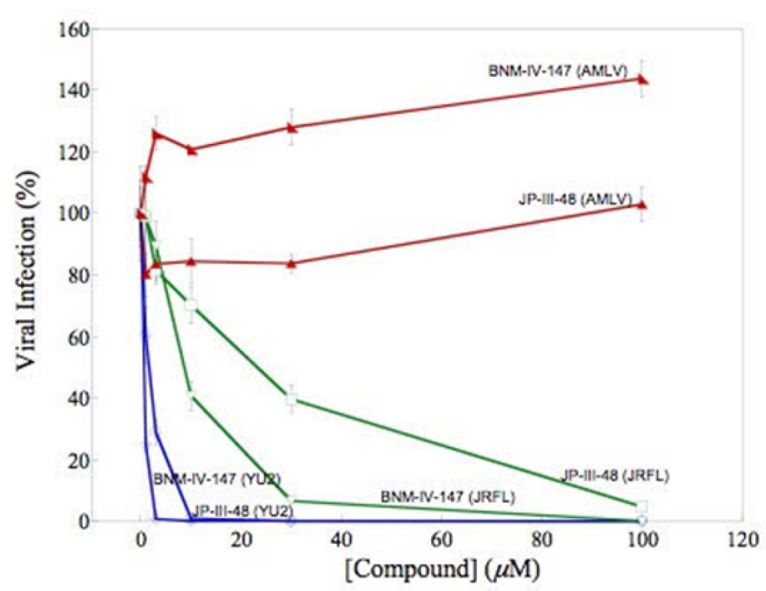

Figure 3. Effect of $(+)-(R, R)-4$ (JP-III-048) and (+)-(R,R)-6 (BNMIV-147) on the infection of Cf2Th-CD4-CCR5 cells by recombinant, luciferase-expressing HIV-1 pseudoviruses carrying envelope glycoproteins of the YU2 or JR-FL strains or that of the amphotropic murine leukemia virus (AMLV, negative control). Virus infection is expressed as the percentage of infection (measured by luciferase activity in the target cells) observed in the presence of increasing concentrations of compound relative to the level of infection observed in the absence of compound.

$\mu \mathrm{M}$; Table 1). Interestingly, however, the binding affinity of $(+)-(R, R)-6$ for monomeric gp120 again appeared to decrease relative to the less potent congeners $(+)-(R, R)-4$ and $(+)-(R, R)-5$. In turn, the diguanidinium congener $(-)-(R, R)-$ 7 , prepared in a similar fashion from $(+)-(R, R)-4$, exhibiting a thermodynamic profile nearly identical to that of $(+)-(R, R)-6$, proved to be approximately half as potent (see Table 1 ). Crystal structures were also determined for gp120 complexes with $(+)-(R, R)-6$ and with $(-)-(R, R)-7$. The binding poses for these (methylguanidino) methyl derivatives are almost the same as those for their (methylamino) methyl congeners, $(+)-(R, R)-4$ and 5 (Figure 4); however, the added guanidine moieties point away from possible hydrogen-bonding partners, including ${ }^{\mathrm{gp} 120}$ Asp 474. For all four complexes, there are hydrogen bonds from the guanidinium group at indane position 1 to the carbonyl groups of ${ }^{9 p 120}$ Met426 and ${ }_{\mathrm{gp} 120}$ Gly431. For $(+)-(R, R)-6$ and $(-)-(R, R)-7$, distances to the carbonyl oxygen of ${ }^{\text {gp } 120} \mathrm{Glu} 429$ suggest additional hydrogen
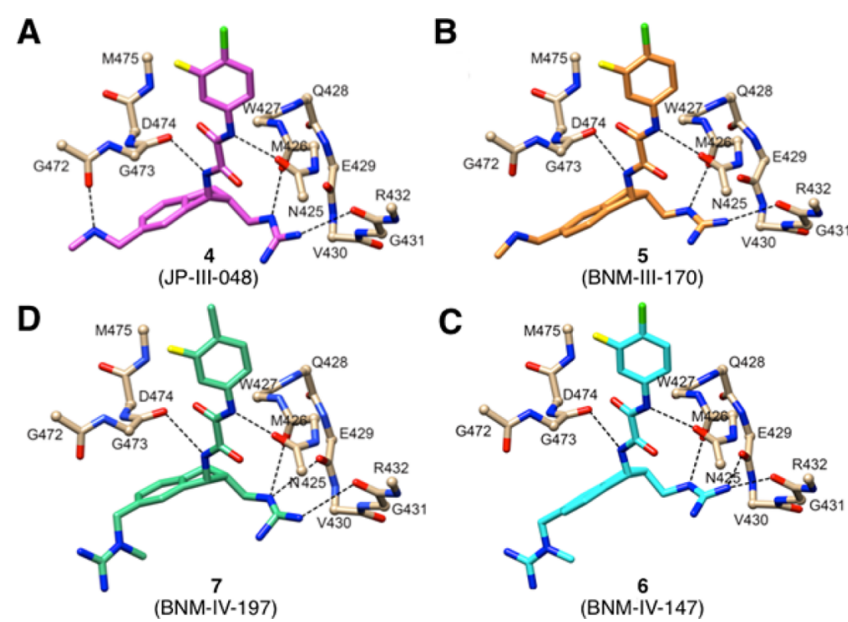

Figure 4. Comparison of gp120-bound poses of 4 (A), 5 (B), 6 (C), and $7(\mathrm{D})$, all $(R, R)$-series. Orientations are the same based on superpositioning of gp $120 \mathrm{C} \alpha$ positions. Hydrogen-bond interactions are shown as dashed lines.

bonding $\left(d_{\mathrm{N}-\mathrm{O}}=3.05 \AA\right.$ for 6 and $3.21 \AA$ for 7 as compared to 3.3-3.4 $\AA$ for 4 and 5).

Although the disparity between potency and protein affinity still remains an unresolved paradox, a possible explanation lies in that thermodynamic measurements employ monomeric gp120, which only comprises part of the fully functional Env (a trimer of gp41-gp120). Indeed, interactions of the more potent inhibitors and the trimeric functional Env might also entail interactions between the Env protomers themselves that in vitro assays employing monomeric gp120 would not reveal. Logically, nonetheless, thermodynamic data are in agreement with crystallographic data, also obtained on monomeric glycoprotein.

In summary, the design of a more potent and more broadly active small molecule CD4-mimetic inhibitor of HIV-1 infection has been achieved exploiting structural and thermodynamic data. In particular, $(+)-(R, R)-6$ exhibited 1 order of magnitude improvement in inhibitory potency, over our last generation of compounds, against the neutralizationresistant HIV-1 strain JR-FL. Virus neutralization strategies employing these new constructs, in addition to direct viral entry inhibition, are currently being explored and will be reported in due course. These strategies rely on the unique ability of the CD4-mimetic inhibitors to expose, upon Env binding, epitopes recognized by antibodies present in HIV-infected individuals. ${ }^{9,10}$ That we have already demonstrated the capability of the earlier congeners of $(+)-(R, R)-6$, namely, 3 and 4 , to enhance the efficacy of immune responses both against the HIV virus itself $^{7}$ and against infected cells ${ }^{10}$ is very encouraging.

\section{ASSOCIATED CONTENT}

\section{Supporting Information}

The Supporting Information is available free of charge on the ACS Publications website at DOI: 10.1021/acsmedchemlett.5b00471.

Synthesis, experimental methods and crystallographic data (PDF)

\section{Accession Codes}

Accession Codes Coordinates and structure factors have been deposited in the Protein Data Bank with the following accession numbers: 5F4L, 5F4P, 5F4R, and 5F4U. 


\section{AUTHOR INFORMATION}

\section{Corresponding Author}

*E-mail: smithab@sas.upenn.edu. Phone: 215-898-4860.

\section{Present Addresses}

J.P.: LG Chem, Daejeon, South Korea.

J.R.C.: Seattle Genetics, Bothell, Washington 98021, United States.

J.M.L.: Teva Pharmaceuticals USA Inc., Horsham, Pennsylvania 19044, United States.

D.J.W.: Max Planck Florida Institute for Neuroscience, Jupiter, Florida 33458, United States.

\section{Author Contributions}

The manuscript was written through contributions of all authors. All authors have given approval to the final version of the manuscript.

\section{Funding}

Funding was provided by a Program Project (NIH GM 56550) to J.L., E.F., W.A.H., A.B.S., and J.S.

\section{Notes}

The authors declare no competing financial interest.

\section{ACKNOWLEDGMENTS}

We thank Irwin Chaiken and all the members of the P01 Consortium Structure-Based Antagonism of HIV-1 Envelope Function in Cell Entry. Dr. Rakesh Kohli, and Drs. George Furst and Jun Gu (University of Pennsylvania) are also acknowledged for their assistance obtaining mass and NMR spectra, respectively.

\section{ABBREVIATIONS}

HIV-1, human immunodeficiency virus type 1; SIV, simian immunodeficiency virus; sCD4, soluble CD4; ITC, isothermal titration calorimetry; A-MLV, amphotropic murine leukemia virus; GMT, geometric mean titer; THF, tetrahydrofuran; Boc, tert-butoxycarbonyl; HMDS, hexamethyldisilazide; DPEN, diphenylethylenediamine; TBS, tert-butyldimethylsilyl; DPPA, diphenylphosphorylazide; DBU, 1,8-diazabicyclo[5.4.0]undec7-ene; TBTU, O-(benzotriazol-1-yl)- $N, N, N^{\prime}, N^{\prime}$-tetramethyluronium tetrafluoroborate; DIPEA, diisopropylethyl amine; TBAF, tributylammonium fluoride; DEAD, diethylazodicarboxylate; TFA, trifluoroacetic acid

\section{REFERENCES}

(1) United Nations. UNAIDS Fact Sheet 2014.

(2) Karim, Q.; Karim, S.; Frohlich, J. Effectiveness and Safety of Tenofovir Gel, an Antiretroviral Microbicide, for the Prevention of HIV Infection in Women. Science 2010, 329, 1168-1174.

(3) Celum, C.; Baeten, J. Tenofovir-Based Pre-Exposure Prophylaxis for HIV Prevention: Evidence and Evolving Questions. Curr. Opin. Infect. Dis. 2012, 25, 51-57.

(4) LaLonde, J. M.; Le-Khac, M.; Jones, D. M.; Courter, J. R.; Park, J.; Schön, A.; Princiotto, A. M.; Wu, X.; Mascola, J. R.; Freire, E.; Sodroski, J.; Madani, N.; Hendrickson, W. A.; Smith, A. B., III Structure-Based Design and Synthesis of an HIV-1 Entry Inhibitor Exploiting X-Ray and Thermodynamic Characterization. ACS Med. Chem. Lett. 2013, 4, 338-343.

(5) Courter, J. R.; Madani, N.; Sodroski, J.; Schön, A.; Freire, E.; Kwong, P. D.; Hendrickson, W. A.; Chaiken, I. M.; LaLonde, J. M.; Smith, A. B., III Structure-Based Design, Synthesis and Validation of CD4-Mimetic Small Molecule Inhibitors of HIV-1 Entry: Conversion of a Viral Entry Agonist to an Antagonist. Acc. Chem. Res. 2014, 47, $1228-1237$.
(6) Zhao, Q.; Ma, L.; Jiang, S.; Lu, H.; Liu, S.; He, Y.; Strick, N.; Neamati, N.; Debnath, A. K. Identification of N-Phenyl-N'-(2,2,6,6tetramethyl-piperidin-4-yl)-oxalamides as a New Class of HIV-1 Entry Inhibitors that Prevent gp120 Binding to CD4. Virology 2005, 339, 213-225.

(7) For recent work by Debnath et al. in the area of HIV entry inhibitors see: Curreli, F.; Kwon, Y.; Zhang, H.; Scacalossi, D.; Belov, D. S.; Tikhonov, A. A.; Andreev, I. A.; Altieri, A.; Kurkin, A. V.; Kwong, P. D.; Debnath, A. K. Structure-Based Design of a Small Molecule CD4-Antagonist with Broad Spectrum Anti-HIV-1 Activity. J. Med. Chem. 2015, 58, 6909-6927.

(8) Curreli, F.; Haque, K.; Xie, L.; Qiu, Q.; Xu, J.; Yong, W.; Tong, X.; Debnath, A. K. Synthesis, Antiviral Activity and Resistance of a Novel Small Molecule HIV-1 Entry Inhibitor. Bioorg. Med. Chem. 2015, 23, 7618 .

(9) Madani, N.; Princiotto, A. M.; Schön, A.; LaLonde, J.; Feng, Y.; Freire, E.; Park, J.; Courter, J. R.; Jones, D. M.; Robinson, J.; Liao, H.X.; Moody, M. A.; Permar, S.; Haynes, B.; Smith, A. B.; Wyatt, R.; Sodroski, J. CD4-Mimetic Small Molecules Sensitize Human Immunodeficiency Virus to Vaccine-Elicited Antibodies. J. Virol. 2014, 88, 6542-6555.

(10) Richard, J.; Veillette, M.; Brassard, N.; Iyer, S. S.; Roger, M.; Martin, L.; Pazgier, M.; Schön, A.; Freire, E.; Routy, J.-P.; Smith, A. B., III; Park, J.; Jones, D. M.; Courter, J. R.; Melillo, B. N.; Kaufmann, D. E.; Hahn, B. H.; Permar, S. R.; Haynes, B. F.; Madani, N.; Sodroski, J. G.; Finzi, A. CD4Mimetics Sensitize HIV-1-Infected Cells to ADCC. Proc. Natl. Acad. Sci. U. S. A. 2015, 112, E2687-E2694.

(11) For efforts by Tamamura et al. toward the optimization of CD4mimetic HIV entry inhibitors, in particular via modification of the socalled Region III, see: Mizuguchi, T.; Harada, S.; Miura, T.; Ohashi, N.; Narumi, T.; Mori, H.; Irahara, Y.; Yamada, Y.; Nomura, W.; Matsushita, S.; Yoshimura, K.; Tamamura, H. A Minimally Cytotoxic CD4Mimic as an HIV Entry Inhibitor. Bioorg. Med. Chem. Lett. 2016, 26, 397-400 and refs cited therein.

(12) Liu, Y.; Schön, A.; Freire, E. Optimization of CD4/gp120 Inhibitors by Thermodynamic-Guided Alanine-Scanning Mutagenesis. Chem. Biol. Drug Des. 2013, 81, 72-78.

(13) Molecular Operating Environment (MOE), 2012.10; Chemical Computing Group Inc.: Montreal, QC, Canada, 2012.

(14) Jones, G.; Willet, P.; Glen, R. C.; Leach, A. R.; Taylor, R. Development And Validation of A Genetic Algorithm For Flexible Docking. J. Mol. Biol. 1997, 267, 727-748.

(15) Verdonk, M. L.; Cole, J. C.; Hartshorn, M. J.; Murray, C. W.; Taylor, R. D. Improved Protein-Ligand Docking Using GOLD. Proteins: Struct., Funct., Genet. 2003, 52, 609-623.

(16) Kwong, P. D.; Wyatt, R.; Robinson, J.; Sweet, R. W.; Sodroski, J.; Hendrickson, W. A. Structure of an HIV gp120 Envelope Glycoprotein in Complex with the CD4 Receptor and a Neutralizing Human Antibody. Nature 1998, 393, 648-659.

(17) Ros, A.; Magriz, A.; Dietrich, H.; Lassaletta, J. M.; Fernández, R. Stereoselective Synthesis of syn $\beta$-Hydroxy Cycloalkane Carboxylates: Transfer Hydrogenation of Cyclic $\beta$-Keto Esters via Dynamic Kinetic Resolution. Tetrahedron 2007, 63, 7532-7537.

(18) Odadzic, D.; Bramsen, J. B.; Smicius, R.; Bus, C.; Kjems, J.; Engels, J. W. Synthesis of 2'-O-Modified Adenosine Building Blocks and Application for RNA Interference. Bioorg. Med. Chem. 2008, 16, 518-529.

(19) Kwon, Y. D.; Finzi, A.; Wu, X.; Dogo-Isonagie, C.; Lee, L. K.; Moore, L. R.; Schmidt, S. D.; Stuckey, J.; Yang, Y.; Zhou, T.; Zhu, J.; Vicic, D. A.; Debnath, A. K.; Shapiro, L.; Bewley, C. A.; Mascola, J. R.; Sodroski, J. G.; Kwong, P. D. Unliganded HIV-1 gp120 Core Structures Assume the CD4-Bound Conformation with Regulation by Quaternary Interactions and Variable Loops. Proc. Natl. Acad. Sci. U. S. A. 2012, 109, 5663-5668. 